Article

\title{
PD Plus Dynamic Pressure Feedback Control for a Direct Drive Stewart Manipulator
}

\author{
Chenyang Zhang \\ School of Mechatronics Engineering, Harbin Institute of Technology, West Dazhi Street No.92, Harbin 150000, \\ China; zhangcyhit@126.com
}

Received: 19 January 2020; Accepted: 26 February 2020; Published: 3 March 2020

\begin{abstract}
In order to ensure good dynamic characteristics, servo valve is usually adopted as the drive part of Stewart manipulator which causes huge power consumption, while direct drive electro-hydraulic servo system has the advantages of energy saving, simple structure, convenient installation, and low failure rate. But its dynamic characteristics are so poor that it can only be applied to occasions where quick response is not needed. On the consideration above, following works are done in this paper. Since current coupling exists in the control system based on the speed of the servo motor as the control input, the control system of the direct drive Stewart manipulator is established based on the current of the servo motor as the control input in which the current coupling can be solved. In order to improve the dynamic characteristics of the direct drive Stewart manipulator, a Proportion Differentiation (PD) plus dynamic pressure feedback control strategy is also put forward in this paper, which is verified by using a simulated hydraulically driven Stewart manipulator. Simulation results show that both dynamic coupling and current coupling are solved and the control strategy proposed in this paper can significantly increase the bandwidths of all degrees of freedom.
\end{abstract}

Keywords: energy saving; direct drive; vector control; Stewart manipulator; modal space; PD control; dynamic pressure feedback

\section{Introduction}

Parallel manipulators are becoming more and more interesting in the field of machine tools and robots due to their precision, stiffness, and dynamics [1-7]. Jiang proposed a modal space control strategy suitable for the electro-hydraulic Steward manipulator [8]. This method maps the control and feedback variables of the joint space to the modal space, therefore every degree of freedom can be individually controlled and adjusted. Compared with the traditional PID controller, simulation results show that the modal space controller has better control performance in almost all aspects.

In order to ensure good dynamic characteristics, servo valve is usually adopted to Stewart manipulator as the drive part, which waste large amount of energy. Direct drive electro-hydraulic servo system has the advantages of energy saving, simple structure, convenient installation, and low failure rate [9]. But its dynamic characteristics are so poor that it can only be applied to occasions where a quick response is not needed. With greatly improved dynamic characteristics, the application of direct drive system in Stewart manipulator instead of valve control system can not only greatly reduce the energy consumption, but also an independent pump station, which is quite big, is no longer needed. For its poor dynamic characteristics, the structure and control strategy of direct drive system are widely studied. Shen et al. [10] enhanced the dynamic response of a new type electro-hydraulic actuator adopted variable displacement pump with a fuzzy controller. Sliding mode controller was also applied to direct drive system [11-13], especially, the real-time values of the rod velocity and acceleration obtained from the measurement signal of displacement were applied with the discrete variable structure 
control algorithm, by using a third-order integral chain differentiator [14]. Wang et al. proposed a state estimation-based model predictive control strategy to deal with the constraints and poor robustness problems existing in the system of a servo motor driven pump-controlled asymmetric cylinder [15]. A model predictive controller applicable to the model of unidirectional proportional pump-controlled asymmetric cylinder was proposed based on model predictive control theory and QPhild quadratic optimization algorithm in order to guarantee its non-overshooting output by Xiong et al. [16].

But in the above papers which focused on the structure and control strategy of direct drive system, one servo motor driving one actuator was basically adopted as the main part of direct drive system, and the load was quite simple, but due to the structure of Stewart manipulator, six servo motors driving six piston actuators is the main part, and the coupling of the dynamic model of the Stewart manipulator in this paper which did not exist and was not considered in the papers above should be considered and solved first. Moreover, the control system design with the speed of the servo motor as the control input is usually adopted and the coupling of the dynamic model of the Stewart manipulator can be easily solved with modal space coordinate transformation similar to the servo valve Stewart manipulator, but this will cause current coupling of all six servo motors, which is difficult to be achieved and controlled with the details below. So the control system design which is closer to the direct drive Stewart manipulator with both the coupling of the dynamic model of the Stewart manipulator and the current coupling of the servo motors solved is of great engineering importance, finally, simple but effective control strategy which can improve the dynamic characteristics is also quite necessary, well these requirements are solved in this paper.

The paper is organized as follows. First the key method to improve the dynamic characteristics of direct drive system is summarized. Then, combined with the structure of Stewart manipulator, the transfer function based on the speed of the servo motor and modal space coordinate transformation is established, and the current coupling of the servo motors is investigated. In next the transfer function based on the current of the servo motor and modal space coordinate transformation in which the current coupling disappears is established. Finally, the PD plus dynamic pressure feedback control strategy is put forward according to the transfer function previously obtained, which is verified by using a simulated hydraulically driven Stewart manipulator.

\section{Introduction to the Stewart Manipulator and the Direct Drive System}

\subsection{Introduction to the Stewart Manipulator}

Figure 1 shows the schematic of the Stewart manipulator in this paper. In this manipulator, the spatial motion of the moving platform is generated by six piston actuators. Each piston actuator consists of two parts connected with a gimbal joint where the coulomb friction and viscous friction exist. The manipulator has a total of six degrees of freedom, including three degrees of freedom of motion and three degrees of freedom of rotation around the axis. The equations of motion of the Stewart manipulator are derived using Kane's method [17] and are written as follows:

$$
M_{t}(q) \ddot{q}+C_{t}(\dot{q}, q) \dot{q}+B_{t}(q) \dot{q}+G_{t}(q)=J_{l q}{ }^{T} f_{a},
$$

where $M_{t}, C_{t}, B_{t}$, and $G_{t}$ are the inertial parameter matrix, the coriolis force parameter matrix, the viscous parameter matrix and the gravity parameter matrix, $J_{l q}^{T}$ is the transpose matrix of $J_{l q^{\prime}} J_{l q}$ is the Jacobin matrix between the work space and the leg, $f_{a}$ is the output force of the piston actuator, $q$ is the six generalized vector of the platform, $q=\left[\begin{array}{lll}x y & y & r x \\ r y & r z\end{array}\right]$. 


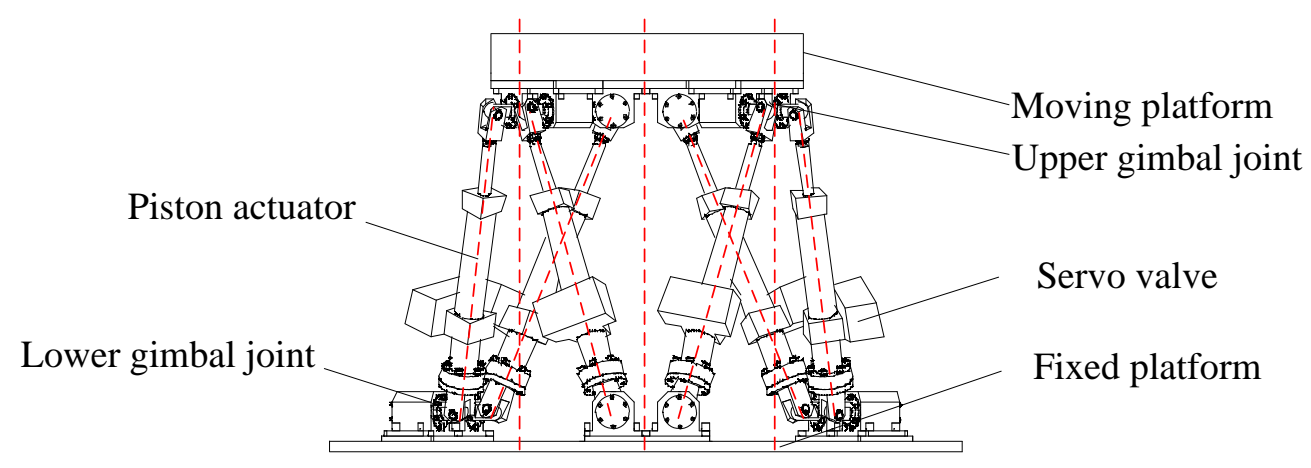

Figure 1. Schematic diagram of a Stewart manipulator.

With movement in small range around the neutral position, Equation (1) is simplified as:

$$
M_{t} \ddot{q}=J_{l q}^{T} f_{a}
$$

for ease of control analysis and design, coulomb, coriolis/centripetal, and gravity terms are omitted. Substituting $J_{l q}^{-1} \ddot{l} \approx \ddot{q}$ into Equation (2), we have:

$$
f_{a}=J_{l q}^{-1 T} M_{t} J_{l q}^{-1 \ddot{l}}
$$

where $\ddot{l}$ is the $6 \times 1$ acceleration vector of the actuator length changes. The mass matrix $M_{a c t}=J_{l q}^{-1 T} M_{t} J_{l q}^{-1}$ is known as the joint space mass matrix. Then Equation (3) is rewritten as:

$$
M_{a c t}^{-1} f_{a}=\ddot{i},
$$

where $M_{\text {act }}^{-1}$ is the inverse matrix of the joint space mass matrix. As modal decomposition can be operated on the matrix above, then $M_{a c t}^{-1}$ can be decomposed as [18]:

$$
M_{a c t}^{-1}=U \Sigma U^{T},
$$

where $\Sigma=\operatorname{diag}\left(\begin{array}{cccc}\sigma_{1} & \sigma_{2} & \cdots & \sigma_{6}\end{array}\right)$ represents the characteristic value, and $U$ is the modal decoupling matrix.

\subsection{Introduction to the Direct Drive System}

Generally a traditional direct drive hydraulic system is shown in Figure 2, the control volume changes as the hydraulic pump rotates reversely, which results in low stiffness, so the dynamic characteristics of this system are poor. The dynamic characteristics of direct drive system will almost reach the same as that of servo valve control system only when the volume of both rod cavity and rod-less cavity are used as the control volume. 


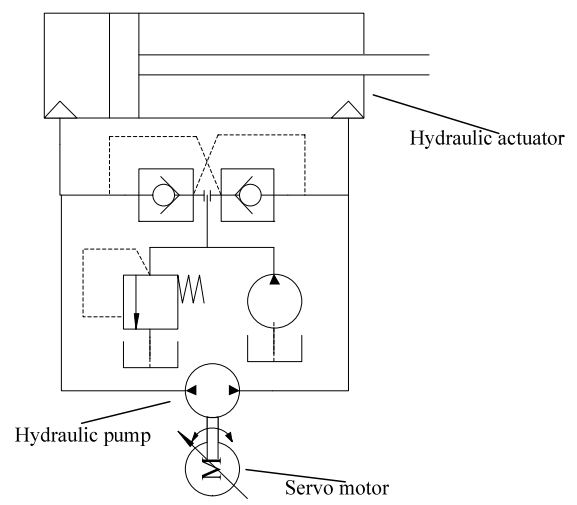

Figure 2. The schematic diagram of a traditional direct drive hydraulic system.

Plummer proposed a direct drive hydraulic system for Stewart manipulator [19]. A modified system which is adopted in this paper is shown in Figure 3. Pressure of the rod cavity and the rod-less cavity is designed the same to compensate the gravity of the manipulator. An energy storage accumulator is set on the other side of the rod-less cavity connected to the motor, and the pressure of the accumulator is set to be the same as that in the rod-less cavity and rod cavity. Therefore, when the manipulator is stationary at the neutral position, the currents in the servo motors are zero. For flow continuity, the displacement ratio of the double pump is set to be the same as the area ratio of the two chambers of the hydraulic actuator. This design also ensures that the speed gain of forward and reverse movement of the hydraulic system is the same, which is convenient for control strategy. Other components in the hydraulic system are mainly used for auxiliary function and have little effect on the dynamic characteristics of the direct drive system. It's that an accumulator is also connected to the rod cavity side of the hydraulic actuator, but this accumulator is isolated to the actuator during the movement, so its influence on dynamics is also ignored.

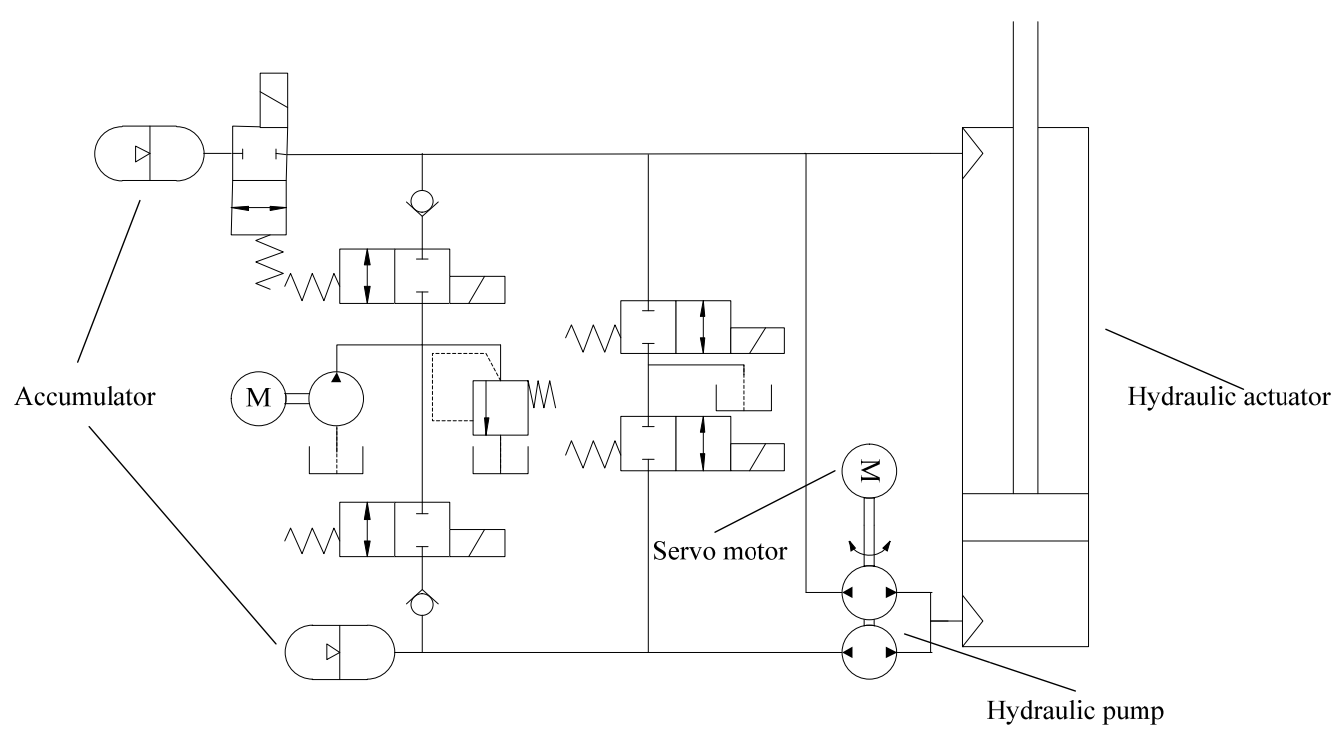

Figure 3. The schematic diagram of the direct drive hydraulic system adopted in this paper.

3. Transfer Function Analysis of the Direct Drive Stewart Manipulator Based on the Motor Speed and Current Coupling

The basic schematic diagram of the direct drive hydraulic system above is shown in Figure 4. 


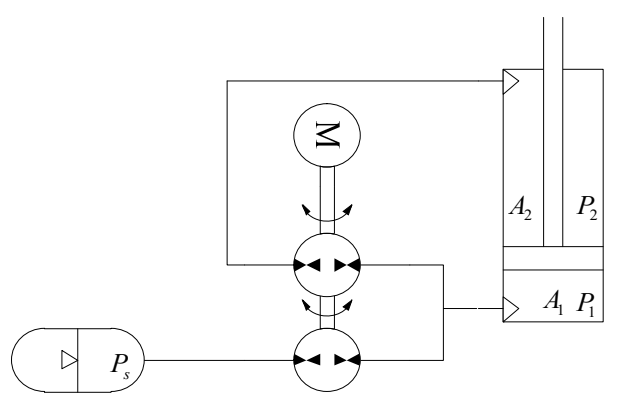

Figure 4. The basic schematic diagram of the direct drive hydraulic system adopted in this paper.

For direct drive system, rotation speed of the servo motor is usually selected as the control input. The flow continuity equation of the rod-less cavity is:

$$
n\left(D_{1}+D_{2}\right) \eta-C_{i p}\left(p_{1}-p_{2}\right)-C_{e p} p_{1}-C_{i m}\left(p_{1}-p_{2}\right)-C_{e m} p_{1}=A_{1} \frac{d l}{d t}+\frac{V_{1}+V_{g}}{\beta_{e}} \frac{d p_{1}}{d t}
$$

where $D_{1}$ and $D_{2}$ are the displacements of the double pump, $A_{1}$ is the piston area of the rod-less cavity, $p_{1}$ and $p_{2}$ are the pressures of the rod cavity and the rod-less cavity, $V_{1}$ is the volume of the rod-less cavity, $V_{g}$ is the volume of the pipeline, $l$ is the displacement of the actuator piston, $\beta_{e}$ is the oil bulk modulus, $C_{i m}, C_{e m}, C_{i p}$, and $C_{e p}$ are the internal and external leakage coefficients of the actuator and the double pump, $\eta$ is the volumetric efficiency of the double pump, $n$ is the rotation speed of the servo motor.

The flow continuity equation of the rod cavity is:

$$
C_{i m}\left(p_{1}-p_{2}\right)-n D_{1} \eta-C_{e m} p_{2}=-A_{2} \frac{d l}{d t}+\frac{V_{2}+V_{g}}{\beta_{e}} \frac{d p_{2}}{d t},
$$

where $A_{2}$ is the piston area of the rod cavity, $V_{2}$ is the volume of the rod cavity.

From the relationship between the double pump and the area ratio of the two chambers of the hydraulic actuator, we know:

$$
A_{2} / A_{1}=D_{1} /\left(D_{1}+D_{2}\right)=e,
$$

so, Equation (7) will be rewritten as:

$$
C_{i m}\left(p_{1}-p_{2}\right)-e n\left(D_{1}+D_{2}\right) \eta-C_{e m} p_{2}=-e A_{1} \frac{d l}{d t}+\frac{V_{2}+V_{g}}{\beta_{e}} \frac{d P_{2}}{d t}
$$

and load pressure $p_{L}$ can be defined as:

$$
p_{L}=\frac{F}{A_{1}}=\frac{P_{1} A_{1}-P_{2} A_{2}}{A_{1}}=P_{1}-e P_{2}
$$

assuming $V_{2}=V_{1}, A_{p}=A_{1}$ and $V_{p}=D_{1}+D_{2}$, writing $V_{t}$ as $\frac{V_{2}+V_{g}}{1+e^{2}}$ for convenience, combining with Equations (6)-(9), the flow continuity equation of the direct drive system can be rewritten as:

$$
n V_{p} \eta-K_{c e} p_{L}-A_{p} \frac{d l}{d t}=\frac{V_{t}}{\beta_{e}} \frac{d p_{L}}{d t}
$$

where $K_{c e}=\frac{1+e}{1+e^{2}} C_{i m}+\frac{C_{i m}+C_{i p}}{1+e^{2}}$.

Taking Laplace transform of Equation (11), the flow equilibrium equation of the $i$-th actuator can be written as:

$$
\eta V_{p} n_{u, j}=l_{j} A_{p} s+\left(\frac{V_{t}}{\beta_{e}} s+K_{c e}\right) p_{L, j}
$$


also, the net output force of the $i$-th actuator is given as:

$$
f_{a, i}=A_{p} P_{L, i}-b_{c} l_{i} s
$$

where $b_{c}$ is the viscous damping coefficient of the actuator. Combining with Equations (13) and (4), we have the dynamic equations in joint space as:

$$
\sum_{j=1}^{6}\left\{M_{a c t}^{-1}(i, j)\left[\frac{h V_{p} n_{j} / A_{p}-s\left(1+\frac{b_{c} K_{c e}}{A_{p}^{2}}+\frac{b_{c} V_{t}}{b_{e} A_{p}^{2}} s\right)}{\frac{V_{t}}{\beta_{e} A_{p}^{2}} s+\frac{K_{c e}}{A_{p}^{2}}}\right]\right\}=l_{i} s^{2},
$$

combining with Equation (5), defining new variables $\tilde{l}=U^{T} l, \tilde{n}_{u}=U^{T} n_{u}$, the dynamic model in the modal space is given by:

$$
s\left[s^{2} \frac{V_{t}}{\beta_{e} A_{p}^{2}} E_{6 \times 6}+s\left(\frac{b_{c} V_{t}}{\beta_{e} A_{p}^{2}} E_{6 \times 6}+\frac{K_{c e}}{A_{p}^{2}} \Sigma\right)+\left(1+\frac{B_{c} K_{c e}}{A_{p}^{2}}\right) \Sigma\right] \tilde{l}=\eta V_{p} \Sigma \tilde{n}_{u} / A_{p},
$$

therefore, similar to the transform function of the servo valve Stewart manipulator, the coupled direct drive system can be decomposed into six independent third-order systems along the modal direction, which consist of an integration link and a two-order oscillation link, which have the same form as a hydraulically driven mechanical system with one degree of freedom.

Considering the torque equation of the servo motor and vector control, following equation can be obtained as:

$$
K_{t} i_{q}=J \frac{d \omega_{m}}{d t}+B \omega_{m}+\frac{V_{p}}{2 \pi} p_{L}
$$

since roughly $p_{L}=J^{T} l, x={ }^{-1} M_{t} J_{l, x}{ }^{-1} \ddot{l}_{i}$, ignoring the influence of the accumulator, and according to modal space coordinate transformation, Equation (16) can be written as:

$$
K_{t} i_{q}=2 \pi J U \frac{d \tilde{n}_{u}}{d t}+2 \pi B U \tilde{n}_{u}+\frac{V_{p}}{2 \pi} J_{l q}^{T-1} M J_{l q}^{-1} U \ddot{\tilde{l}}
$$

since the matrix $U$ and $J_{l q}^{T-1} M_{t} J_{l q}^{-1} U$ of the right side are not diagonal, it can be seen that strong coupling still exists in the current of the six servo motors, which not only leads to high requirements for the motor control, but also makes the control of speed extremely difficult. Moreover, the current control signal of each servo motor usually comes from the speed error signal itself, so the strong coupling in the current which cannot be compensated from the speed error signal can greatly reduce the bandwidth of the speed loop of all six servo motors.

Therefore, it is not appropriate to treat the rotation speed as the control input to establish the transfer function of the direct drive Stewart manipulator. However, the electrical mechanical parameters of the motor-gear pump unit are basically stable. Besides, the dynamic characteristics of the current-loop of the servo motor is satisfactory, which can be guaranteed from the electrical time constant of $1 \mathrm{kw}-2 \mathrm{kw}$ servo motor, which is usually about $3.5 \mathrm{~ms}$. Therefore, the control system of the direct drive Stewart manipulator with the current of the servo motor as the control input will be closer to the actual system, and the control algorithm based on the current of all six servo motors will bring out actual control effect. Moreover, pre-multiplying both sides of Equation (17) by $U^{T}$, the matrix $U$ and $J_{l q}^{T-1} M_{t} J_{l q}^{-1} U$ of right side become diagonal, which means the coupling in current can be solved with modal space coordinate transformation. 
4. Transfer Function of the Direct Drive Stewart Manipulator Based on the Motor Current and Modal Space Coordinate Transformation

Taking Laplace transform, Equation (16) can be rearranged as:

$$
\omega_{m}=\frac{K_{t} i_{q}-T_{L}}{J s+B}
$$

where $T_{L}$ is the load torque $T_{L}=\frac{\left(p_{L}-e \Delta p_{s}\right) V_{p}}{2 \pi}, \Delta p_{s}$ is the pressure change of the accumulator, if $\Delta p_{s}$ can be ignored, the load torque $T_{L}$ can be defined as:

$$
T_{L}=\frac{p_{L} V_{p}}{2 \pi}
$$

Equation (19) can be rewritten as:

$$
n=\frac{K_{t e} i_{q}-p_{L} V_{p e}}{J s+B}
$$

where $K_{t e}=\frac{K_{t}}{2 \pi}, V_{p e}=\frac{V_{p}}{4 \pi^{2}}$.

The relationship between the pressure change of the accumulator $\Delta p_{s}$ and the displacement of the actuator is:

$$
\Delta p_{s}=p_{s}-p_{1}\left(p_{2}\right)=K_{x} l
$$

where $K_{x}=\frac{k p_{a_{0}} V_{a_{0}}^{k}}{4 \pi V_{a_{0}}^{k+1} A_{p}}$ is the equivalent spring stiffness. Taking Laplace transform, the flow equilibrium equation of the $i$-th actuator can be written as:

$$
V_{p} \eta K_{t e} i_{q}-\left[J A_{p} s^{2}+B A_{p} s+V_{p} \eta K_{x}\right] l=\left[J \frac{V_{t}}{\beta_{e}} s^{2}+\left(B \frac{V_{t}}{\beta_{e}}+J K_{c e}\right) s+\left(B K_{c e}+V_{p e} V_{p} \eta\right)\right] p_{L, j}
$$

Equation (22) can be rewritten as:

$$
p_{L, j}=\frac{V_{p} \eta K_{t e} i_{q}-\left[J A_{p} s^{2}+B A_{p} s+V_{p} \eta K_{x}\right] l_{j}}{\left[J \frac{V_{t}}{\beta_{e}} s^{2}+\left(B \frac{V_{t}}{\beta_{e}}+J K_{c e}\right) s+\left(B K_{c e}+V_{p e} D_{h} \eta\right)\right]}
$$

therefore, although the direct drive system in this paper also treat the current as the control input, its transfer function is different from that of a servo valve controlled system.

Similar to Equation (14), we have the dynamic equations of the direct drive Stewart manipulator in joint space as:

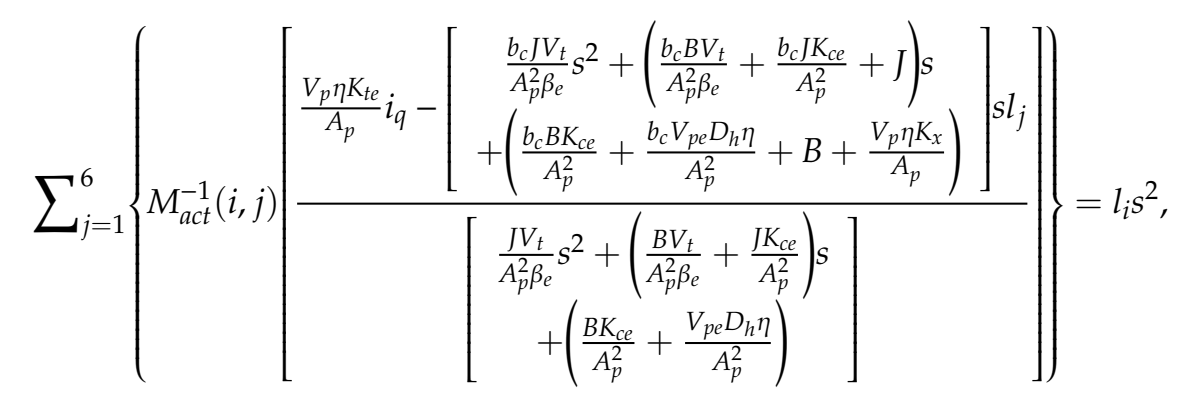


similarly, the transfer function of the direct drive system based on modal space coordinate transformation can be written as:

$$
\left\{\begin{array}{c}
\frac{J V_{t}}{A_{p}^{2} \beta_{e}} E_{6 \times 6} S^{3}+\left[\left(\frac{B V_{t}}{A_{p}^{2} \beta_{e}}+\frac{J K_{c e}}{A_{p}^{2}}\right) E_{6 \times 6}+\sum \frac{b_{c} J V_{t}}{A_{p}^{2} \beta_{e}}\right] s^{2} \\
+\left[\left(\frac{B K_{c e}}{A_{p}^{2}}+\frac{V_{p e} V_{p} \eta}{A_{p}^{2}}\right) E_{6 \times 6}+\Sigma\left(\frac{b_{c} B V_{t}}{A_{p}^{2} \beta_{e}}+\frac{b_{c} J K_{c e}}{A_{p}^{2}}+J\right)\right] s \\
+\Sigma\left(\frac{b_{c} B K_{c e}}{A_{p}^{2}}+\frac{b_{c} V_{p e} V_{p} \eta}{A_{p}^{2}}+B\right)
\end{array}\right\} s \tilde{l}+\Sigma \frac{V_{p} \eta K_{x}}{A_{p}} \tilde{l}=\Sigma \frac{V_{p} \eta K_{t e}}{A_{p}} \tilde{i}_{q},
$$

in this way, the transfer function of the direct drive Stewart manipulator is obtained.

It is worth noting that the elasticity of the accumulator will affect the basic structure of the transfer function. If the elasticity of the accumulator needs to be considered, the system is composed of two second-order oscillation links. But if the accumulator in the system has is so large that the elasticity of the accumulator can be ignored that is the displacement of the piston rod will not cause an apparent change of the pressure in the accumulator, then correspondingly, the transfer function consists of an integral link, an inertial link, and a second-order oscillation link.

According to the consideration above, a large accumulator should be selected. Although the order of the transfer function can not be reduced, for control algorithm, the less complex the transfer function, the better control effect can be easily achieved. Generally, the leakage is little, $K_{c e}$ can be omitted, so Equation (25) becomes:

$$
\left\{\begin{array}{l}
\frac{J V_{t}}{\beta_{e} A_{p}^{2}} E_{6 \times 6} S^{3}+\left[\Sigma \frac{b_{c} J V_{t}}{\beta_{e} A_{p}^{2}}+\frac{B V_{t}}{A_{p}^{2} \beta_{e}} E_{6 \times 6}\right] s^{2}+\left[\frac{V_{p e} V_{p} \eta}{A_{p}^{2}} E_{6 \times 6}+\Sigma\left(J+\frac{b_{c} B V_{t}}{A_{p}^{2} \beta_{e}}\right)\right] s \\
+\Sigma\left(\frac{b_{c} V_{p e} V_{p} \eta}{A_{p}^{2}}+B\right)
\end{array}\right\} s \tilde{l}=\Sigma \frac{V_{p} \eta K_{t e}}{A_{p}} \tilde{i}_{q},
$$

while control strategy will be added next.

\section{PD Plus Dynamic Pressure Feedback Control Strategy}

For servo valve Stewart manipulator, the damping ratio of the second-order link can be increased or decreased by using dynamic pressure feedback control [18]. As second-order link still exists, dynamic pressure feedback control is still necessary. Since the inertia link will cause lag, which adversely affects the dynamic characteristics of the manipulator, the PD plus dynamic pressure feedback control strategy is needed for this system.

With dynamic pressure feedback control, Equation (23) will become:

$$
V_{p} \eta K_{t e} i_{q}-\left[J A_{p} s^{2}+B A_{p} s\right] l=\left[J \frac{V_{t}}{\beta_{e}} s^{2}+\left(B \frac{V_{t}}{\beta_{e}}+J K_{c e}\right) s+\left(B K_{c e}+V_{p e} V_{p} \eta\right)\right] p_{L}+G(s) p_{L}
$$

where $G(s)$ is the transfer function of the dynamic pressure feedback link. Therefore, Equation (26) will become:

$$
\left\{\begin{array}{l}
\frac{J V_{t}}{\beta_{e} A_{p}^{2}} E_{6 \times 6} s^{3}+\left[\Sigma \frac{b_{c} J V_{t}}{\beta_{e} A_{p}^{2}}+\frac{B V_{t}}{A_{p}^{2} \beta_{e}} E_{6 \times 6}\right] s^{2} \\
+\left[\frac{V_{p e} V_{p} \eta}{A_{p}^{2}} E_{6 \times 6}+G(s) E_{6 \times 6}+\Sigma\left(J+\frac{b_{c} B V_{t}}{A_{p}^{2} \beta_{e}}\right)\right] s+\Sigma\left(\frac{b_{c} V_{p e} V_{p} \eta}{A_{p}^{2}}+B\right)
\end{array}\right\} s \tilde{l}=\Sigma \frac{V_{p} \eta K_{t e}}{A_{p}} \tilde{i}_{q},
$$

supposing $s+b$ is the denominator of the inertial link and $s^{2}+a_{1} s+a_{2}$ is the denominator of the second-order oscillation link. Denominator of the whole link can be expressed as:

$$
s^{3}+\left(a_{1}+b\right) s^{2}+\left(a_{2}+a_{1} b\right) s+a_{2} b,
$$


since $a_{2}$ is much bigger than $b$ and $a_{1}$ in value, which can be calculated from parameters. So if $G(s)$ is applied as a first derivative link with a proportionality factor and added to the original direct drive system, the damping ratio of the second-order link could be changed, and the oscillation frequency of the second-order link is not greatly affected, but the coefficient of the inertia link is affected. So in order to achieve the corresponding increase or decrease of the damping ratio, the first derivative of the load pressure should be taken, similar to the servo valve Stewart manipulator.

Due to the inertia link, the motor-gear pump unit can not quickly convert the error signal into effective flow. Generally, the transfer function of PD link can be written as:

$$
u(x)=K_{p}\left(e(t)+T_{d} \frac{d e(t)}{d t}\right)
$$

Since the differentiation of the error signal can predict its variation, and the change of the input signal also can be obtained from the differentiation of the error signal and added to the control signal for compensation. So PD control should be applied to improve the transient process of the system. Due to the order of Equation (26), the closed-loop poles of the transfer function are basically unchanged with PD control added., but PD control leads to another open-loop zero point which usually has an effect on the damping ratio [20]. So PD control should be used in conjunction with dynamic pressure feedback control to achieve good control effect.

\section{Simulation and Comparison}

The control strategy proposed in this paper is verified by using MATLAB software, the Stewart manipulator and the corresponding control algorithm are both built using Simulink block. The schematic diagram of the simulation model is shown in Figure 5.

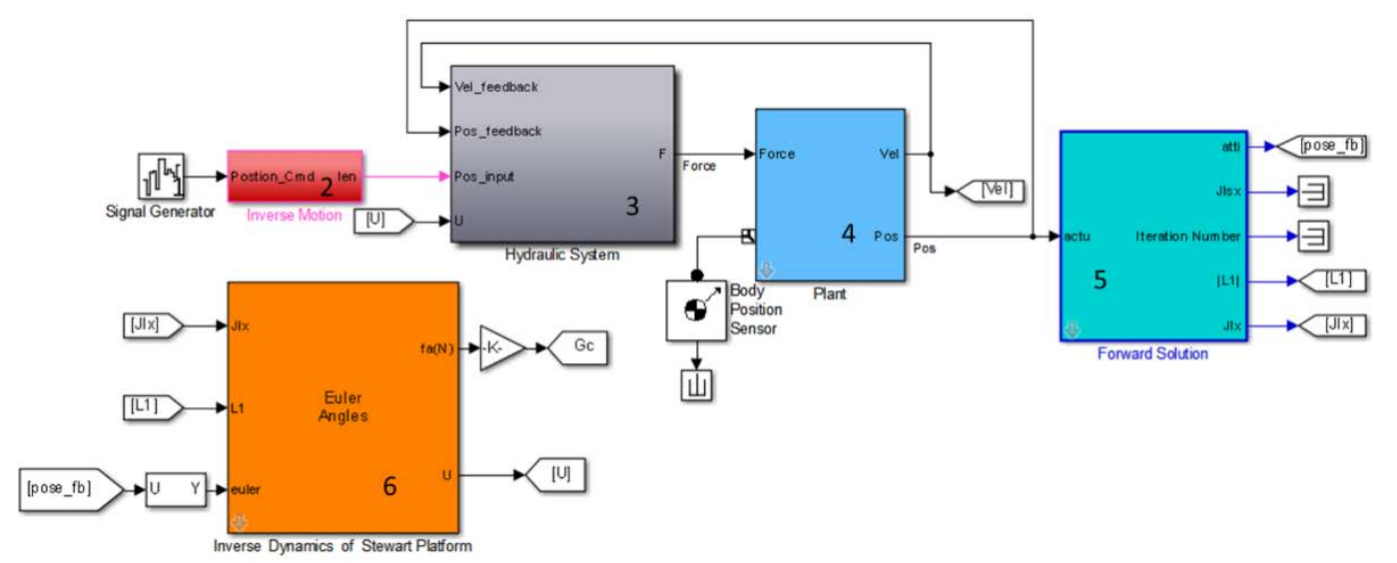

Figure 5. The schematic diagram of the simulation model.

This model consists of six blocks on top level. The first is the Signal Generator block, which can generate white noise signals. The second is the Inverse Motion block, used to calculate actuators commands. The third is the Hydraulic System block, including a PD control block, a Dynamic Pressure Feedback block, and an Actuator block. The fourth is the Plant block, representing the dynamic equations of Stewart manipulator. The fifth is the Forward Solution block, used to compute platform positions from measured actuator lengths in real time. The sixth is the Inverse Dynamics block, which calculates the modal transform matrix. Parameters of the hydraulic system of the simulation model are shown in Table 1. 
Table 1. Parameters of the hydraulic system of the simulation model.

\begin{tabular}{cc}
\hline Descriptions & Values \\
\hline Moment of inertia of the motor-gear pump unit $\left(\mathrm{kg} \cdot \mathrm{m}^{2}\right)$ & $4.1085 \times 10^{-4}$ \\
Viscous coefficient of the motor-gear pump unit $\left(\mathrm{N} \mathrm{m} /\left(\mathrm{rad} \mathrm{s}^{-1}\right)\right)$ & $6.5280 \times 10^{-4}$ \\
Torque coefficient $(\mathrm{N} \cdot \mathrm{m} / \mathrm{A})$ & 1.05 \\
Piston area $\left(\mathrm{m}^{2}\right)$ & $1.3 \times 10^{-3}$ \\
Volumetric efficiency of the double pump & 0.95 \\
Oil bulk modulus (Mpa) & 700 \\
Displacement of the double pump $\left(\mathrm{m}^{3}\right)$ & $0.25 \times 10^{-6}$ \\
\hline
\end{tabular}

According to the principle of modal space coordinate transformation, the displacement signal of the actuator with respect to the position and attitude of the manipulator and the load pressure need to be subjected to the modal space first. Then, the sum of the current signals calculated from the PD control block and the Dynamic Pressure Feedback block needs to be transformed in an inverse transformation as shown in Figure 6.

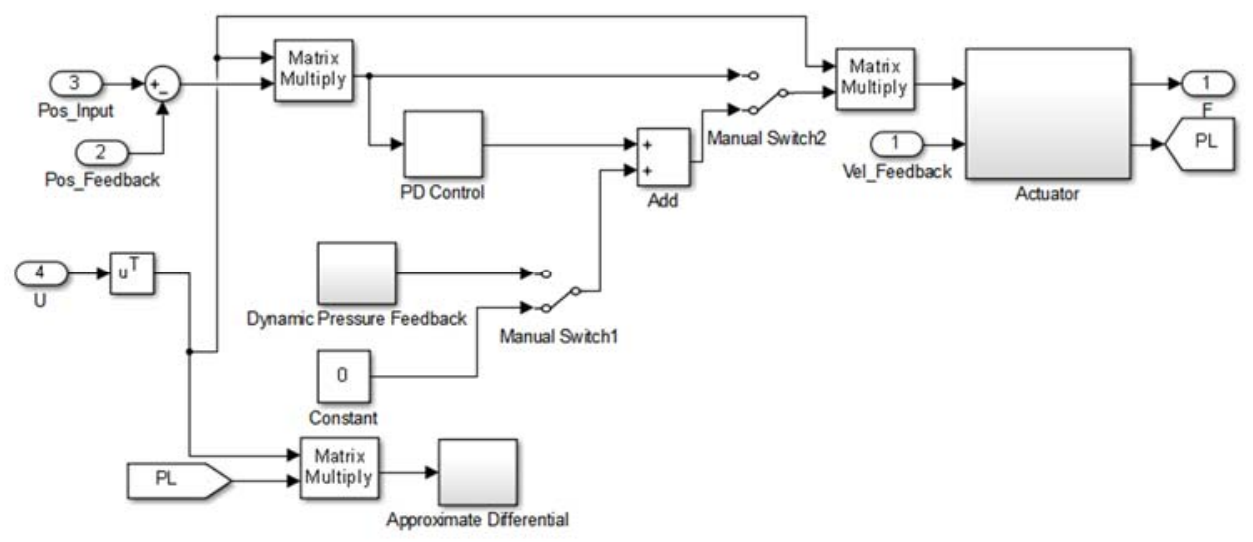

Figure 6. Model diagram of the Hydraulic System block.

In Figure 6, approximate differentiation is adopted in the Approximate Differential block. This approximate differentiation not only ensures the dynamic characteristics of the differentiation, but also reduces the effect of noise. Then, these signals are amplified in the Dynamic Pressure Feedback block with corresponding coefficients. The PD Control block performs proportion and differentiation operation on the error signal. The Actuator block is used to imitate the hydraulic system. Manual Switch blocks (Manual Switch 1 and Manual Switch 2) are also applied to implement different control effects.

According to the previous description, PD control has an impact on the damping ratio of the second order link. It is worth noting that dynamic pressure feedback control will also affect the control effect of PD control, so the parameters of the PD control block and the Dynamic Pressure Feedback block should be adjusted repeatedly to achieve good effect. The closed-loop Bode diagram of the original direct drive Stewart manipulator is shown in Figure 7. Apparently, only four lines can be found, the main reason is that the closed-loop characteristics of $x$ and $y$ direction, rx and ry direction, are basically the same respectively due to the structural symmetry of the manipulator. And both coupling of the dynamic model of the Stewart manipulator and current coupling of the six servo motors are solved and the closed-loop bode diagram of each degree of freedom is independent from each other which is of great engineering importance. And as can be seen in the figure, bandwidths of all six degrees of freedom of the manipulator are narrow. PD control should be added. 


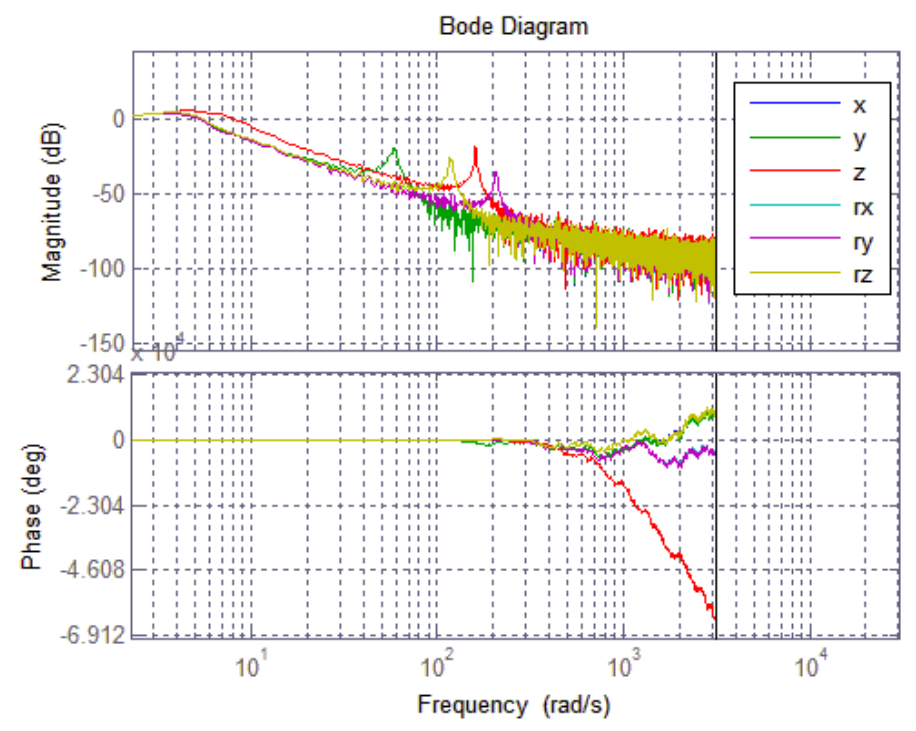

Figure 7. The closed-loop Bode diagram of the original direct drive Stewart manipulator.

The closed-loop Bode diagram of the direct drive Stewart manipulator with PD control is shown in Figure 8. As PD control added, each closed-loop bandwidth is greatly expanded, and closed-loop bandwidths of all six degrees of freedom exceed $20 \mathrm{rad} / \mathrm{s}$, while resonance peaks still exist, and nearly all resonance peaks go through the zero-decibel line which is bad to the system stability. So dynamic pressure feedback control should be applied to increase the damping ratio of the second-order oscillation link.

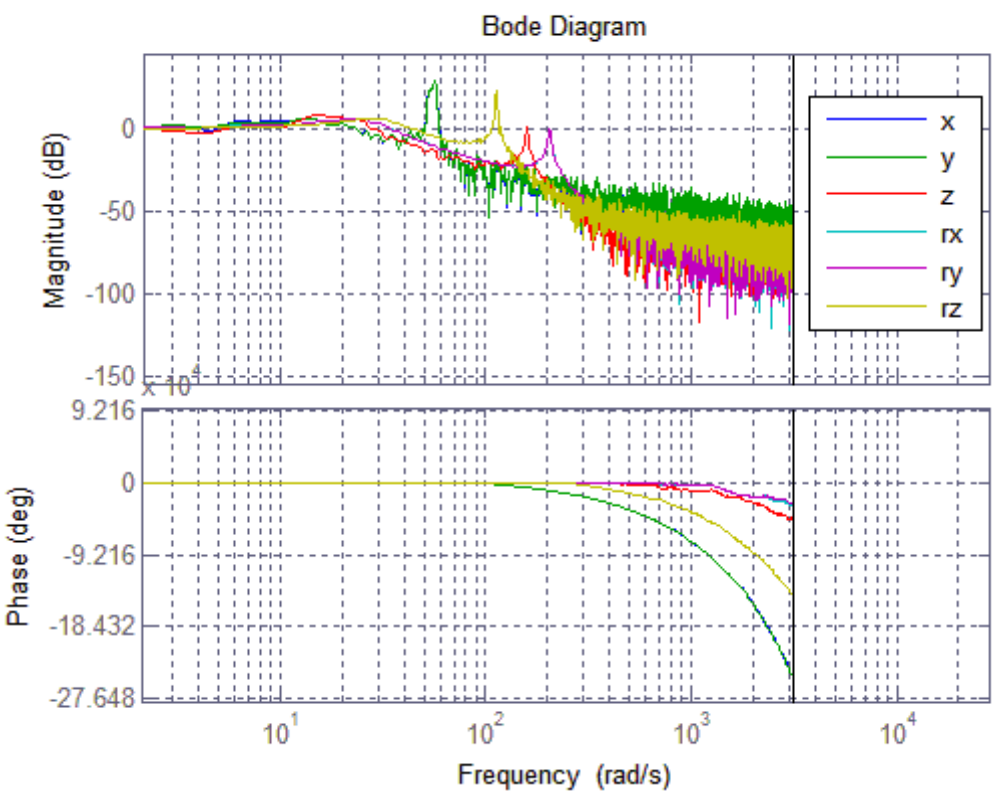

Figure 8. The closed-loop Bode diagram of the direct drive Stewart manipulator with PD control.

The closed-loop Bode diagram of the direct drive Stewart manipulator with PD plus dynamic pressure feedback control is shown in Figure 9. Compared with Figure 8, resonance peaks of all degrees of freedom disappear, and the stability of the direct drive Stewart manipulator is guaranteed. 


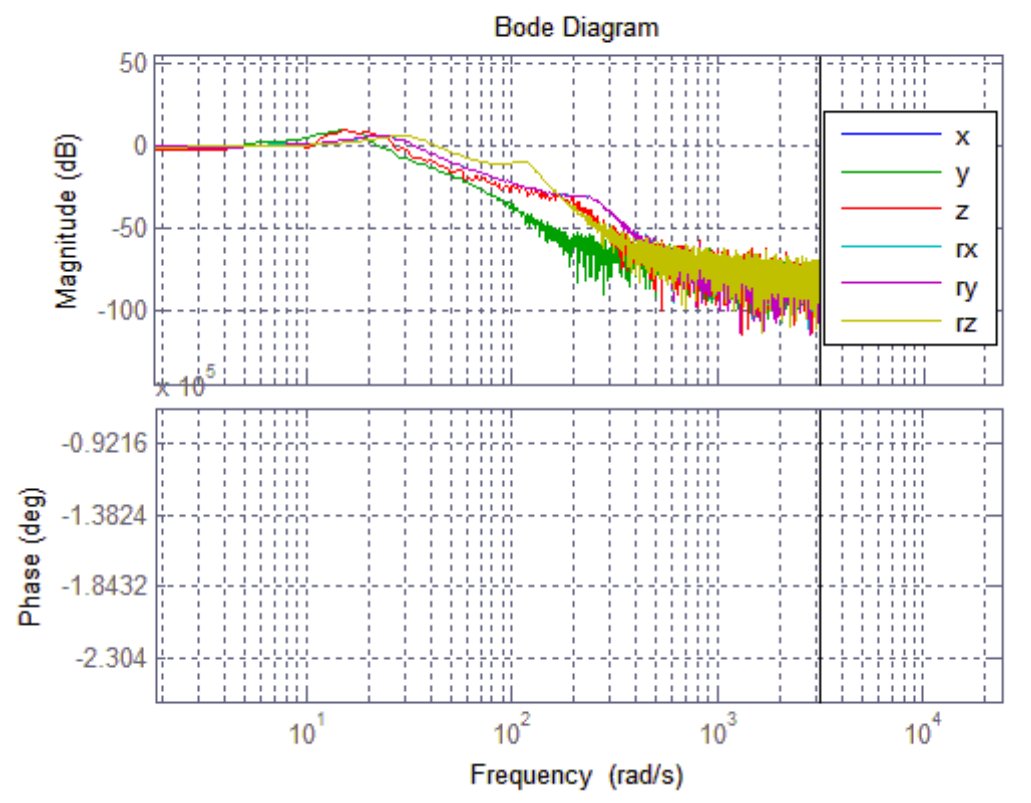

Figure 9. The closed-loop Bode diagram of the direct drive Stewart manipulator with PD plus dynamic pressure feedback control.

\section{Conclusions}

The conclusions organized are as follow:

Modal space coordinate transformation is applicable and quite necessary to the direct drive Stewart manipulator.

The control design based on the speed of the servo motor and modal space coordinate transformation for the direct drive Stewart manipulator can not only solve the dynamic coupling but also cause current coupling which is quite difficult to achieve, while the control design based on the current of the servo motor and modal space coordinate transformation can solve both dynamic coupling and current coupling.

The PD plus dynamic pressure feedback control strategy proposed in this paper is quite simple and effective which can expand the bandwidths of all degrees of freedom of the direct drive Stewart manipulator.

Funding: This research received no external funding

Conflicts of Interest: The authors declare no conflict of interest.

\section{References}

1. Zhang, L.P.; Cong, D.C.; Yang, Z.D.; Zhang, Y.Y.; Han, J.W. Robust tracking and synchronization of double shaking tables based on adaptive sliding mode control with novel reaching law. IEEE Access 2016, 4, 8686-8702. [CrossRef]

2. Zhang, L.P.; Yang, Y.; Yang, C.F.; Han, J.W. Research on the degree of freedom control strategy for redundant-drive shaking tables. In Proceedings of the Institution of Mechanical Engineers Part I Journal of Systems and Control Engineering, Harbin, China, 5-7 August 2015; pp. 488-492. [CrossRef]

3. Zhang, L.P.; Cong, D.C.; Yang, Z.D.; Yang, C.F.; Han, J.W. Attitude synchronous tracking control of double shaking tables based on hybrid fuzzy logic cross-coupled controller and adaptive inverse controller. J. Intell. Fuzzy Syst. 2015, 29, 2537-2546. [CrossRef]

4. Gao, C.H.; Zheng, S.T.; Cong, D.C.; Han, J.W.; Yang, Z.D.; Sun, J.Y. Modeling and control of the cscec multi-function testing system. J. Earthq. Eng. 2018, 2, 257-280. [CrossRef]

5. Gao, C.H.; Cong, D.C.; Liu, X.C.; Yang, Z.D.; Tao, H. Hybrid position/force control of 6-dof hydraulic parallel manipulator using force and vision. Ind. Robot 2016, 43, 274-283. [CrossRef] 
6. Tao, H.; Qu, Z.Y.; Cong, D.C. Hybrid Position/Force Control Scheme for Hydraulic Parallel Manipulator. Nongye Jixie Xuebao 2018, 49, 361-366. [CrossRef]

7. Zeng, D.S.; Yang, Y.; Cong, D.C.; Yang, C.F. Space end effector capturing hybrid simulation system. In Proceedings of the 2015 International Conference on Fluid Power and Mechatronics (FPM), Harbin, China, 5-7 August 2015; pp. 448-453. [CrossRef]

8. Jiang, H.Z.; He, J.F.; Tong, Z.Z. Modal space control for a hydraulically driven stewart platform. JCET 2012, 3, 106-115.

9. Minav, A.M.; Laurila, L.I.E.; Pyrhonen, J.J. Analysis of electro-hydraulic lifting system's energy efficiency with direct electric drive pump control. Autom. Constr. 2013, 30, 144-150. [CrossRef]

10. Shen, W.; Mai, Y.F.; Su, X.Y.; Zhao, J.B.; Jiang, J.H. A new electric hydraulic actuator adopted the variable displacement pump. Asian J. Control 2016, 18, 178-191. [CrossRef]

11. Hu, X.; Al-Ani, D.; Habibi, S. A new Sliding Mode Controller for Electro-Hydraulic Actuator (EHA) applications. In Proceedings of the 2015 International Workshop on Recent Advances in Sliding Modes (RASM 2015), Istanbul, Turkey, 9-11 April 2015. [CrossRef]

12. Li, J.; Gao, Q.; Hou, Y.L.; Sun, Z.; Jia, L.T.; Li, K. Fractional neural sliding mode control for the electro-hydraulic servo system. In Proceedings of the Advanced Information Technology, Electronic \& Automation Control Conference, Chongqing, China, 19-20 December 2015; pp. 810-815. [CrossRef]

13. Zad, H.S.; Ulasyar, A.; Zohaib, A. Robust Model Predictive position Control of direct drive electro-hydraulic servo system. In Proceedings of the International Conference on Intelligent Systems Engineering, Islamabad, Pakistan, 15-17 January 2016. [CrossRef]

14. Jiang, J.H.; Ge, Z.H.; Yang, C.; Liang, H.J. Differentiator-based discrete variable structure controller for direct drive electro-hydraulic servo system. Jilin Daxue Xuebao Gongxueban 2018, 48, 1492-1499. [CrossRef]

15. Wang, X.; Tao, J.F.; Zhang, F.R.; Wu, Y.J.; Liu, C.L. Precision position control of pump-controlled asymmtric cylinder. Zhejiang Daxue Xuebao Gongxueban 2016, 50, 597-602. [CrossRef]

16. Xiong, Z.L.; Tao, J.F.; Zhang, F.R.; Liu, C.L. A model predictive control strategy of pump-controlled asymmetric cylinder using state estimation. Xi'an Jiaotong Daxue Xuebao 2016, 51, 109-115. [CrossRef]

17. Tian, T.X.; Jiang, H.Z.; Tong, Z.Z.; He, J.F.; Huang, Q.T. An inertial parameter identification method of eliminating system damping effect for a six-degree-of-freedom parallel manipulator. Chin. J. Aeronaut. 2015, 28, 582-592. [CrossRef]

18. Tian, T.X.; Jiang, H.Z.; Huang, Q.T.; He, J.F.; Nie, B.X. Control strategy of modal space for a hydraulically-driven stewart platform considering passive joint damping. Huanan Ligong Daxue Xuebao Ziran Kexueban 2015, 43, 56-62. [CrossRef]

19. Cleasby, K.G.; Plummer, A.R. A novel high efficiency electro-hydrostatic flight simulator motion system. Fluid Power Motion Control 2008, 437-449.

20. Hu, S.S. Zi Dong Kong Zhi Yuan Li, 5th ed.; Science Press: Beijing, China, 2010; pp. 163-164. 